\title{
Irritable bowel syndrome-like symptoms associated with endoscopic activity predict ulcerative colitis relapse in patients with clinical remission
}

\author{
Nobuhiko Fukuba $^{1}$, Shunji Ishihara ${ }^{1,2}$, Kousaku Kawashima ${ }^{1,2}$, Yoshiyuki Mishima ${ }^{1}$, Naoki Oshima ${ }^{1}$, \\ Yoshikazu Kinoshita ${ }^{1}$ \\ ${ }^{1}$ Department of Internal Medicine II, Shimane University Faculty of Medicine, Izumo, ${ }^{2}$ IBD Center, Shimane University Hospital, Izumo, Japan
}

Previous studies have shown that symptoms resembling IBS, a functional intestinal disorder characterized by abdominal pain or discomfort along with bowel habit alterations, are often noted by patients with UC in remission. ${ }^{1}$ Furthermore, we recently reported that the prevalence of IBS-like symptoms in patients with UC in clinical remission evidence was $26.7 \%$, which was significantly higher than that in healthy controls $(4.8 \%){ }^{2}$ However, the relapse-free period of patients with UC in remission with IBS-like symptoms has been scarcely investigated.

We examined the records of patients with UC in remission with or without IBS-like symptoms using the subject cohort enrolled in our previous investigation, which was prospectively conducted at a single university and 3 general hospitals in Japan from May 2011 to February $2012 .^{2}$ In that study, patients with UC ( $\geq 18$ years old) in good general health were enrolled by their physicians, while those with hematochezia, history of colectomy, or CRP levels $>0.5 \mathrm{mg} / \mathrm{dL}$ were excluded. The diagnosis of IBS was evaluated by questionnaire results according to the Rome III criteria, and clinical remission was determined based on the clinical activity index (CAI) score ( $\leq 4$ for at least 6 months). ${ }^{3}$ We performed colo-

Received September 30, 2016. Revised December 14, 2016. Accepted February 23, 2017.

Correspondence to Shunji Ishihara, Department of Internal Medicine II, Shimane University Faculty of Medicine, 89-1 Enya-cho, Izumo 693-8501, Japan. Tel: +81-853-20-2190, Fax: +81-853-20-2187,E-mail: si360405@ med.shimane-u.ac.jp

Financial support: None. Conflict of interest: None. noscopy examinations of patients in clinical remission at the same time as the presence or absence of IBS-like symptoms was assessed, while endoscopic activity was evaluated using the Matts grading system. ${ }^{4}$ Endoscopic findings were interpreted by endoscopists who were blinded to the case details. We analyzed patients in clinical remission, with or without IBS-like symptoms, who were available for follow-up examinations every few months. Relapse was defined as CAI $\geq 5$ and the relapse-free periods were assessed using the KaplanMeier method. Patients with interrupted treatment or who were transferred during the study period were considered censored at the time of last follow-up. The relapse-free periods were compared between patients with and without IBSlike symptoms and evaluated using a log-rank test. $P$-values $<0.05$ were considered significant. All calculations were performed using SPSS version 19.0 (IBM Corp., Armonk, NY, USA).

A total of 172 patients were enrolled, of whom 46 (26.7\%) met the Rome III criteria for the presentation of IBS-like symptoms, while the remaining 126 (73.3\%) were without symptoms. ${ }^{2}$ Of those, 68 patients (IBS-like symptom-positive, 21; symptom-negative, 47) completed follow-up examinations during the study period. The baseline characteristics of those subjects are shown in Table 1. Our results showed that CRP levels were not different between the IBS-like symptompositive and symptom-negative groups. On the other hand, endoscopic activity was significantly higher in patients with IBS-like symptoms as compared to those without IBS-like symptoms $(P=0.04)$ (Table 1). The mean relapse-free period

\footnotetext{
๑ Copyright 2017. Korean Association for the Study of Intestinal Diseases. All rights reserved.

This is an Open Access article distributed under the terms of the Creative Commons Attribution Non-Commercial License (http://creativecommons.org/licenses/by-nc/4.0)

which permits unrestricted non-commercial use, distribution, and reproduction in any medium, provided the original work is properly cited.
} 
Table 1. Baseline Characteristics of the Patients

\begin{tabular}{lccc}
\hline \multirow{2}{*}{ Characteristic } & \multicolumn{2}{c}{ IBS-like symptoms } & P-value \\
\cline { 2 - 3 } & Negative & Positive & \\
\hline Number & 47 & 21 & - \\
Sex (male/female) & $23 / 24$ & $15 / 6$ & $0.99^{\mathrm{a}}$ \\
Age (yr) & 50.2 & 43.5 & $0.04^{\mathrm{b}}$ \\
Disease duration (yr) & 8.3 & 7.8 & 0.22 \\
Classification of lesion & & & $0.75^{\mathrm{a}}$ \\
Extensive & $36(17 / 47)$ & $38(8 / 21)$ & \\
Left side & $28(13 / 47)$ & $38(8 / 21)$ & \\
Proctitis & $36(17 / 47)$ & $19(4 / 21)$ & \\
Others & $0(0 / 47)$ & $5(1 / 21)$ & \\
5-ASA or SASP & $89(42 / 47)$ & $86(18 / 21)$ & 0.91 \\
Steroid & $17(8 / 47)$ & $38(8 / 21)$ & $0.99^{\mathrm{a}}$ \\
Immune modulators & $15(7 / 47)$ & $38(8 / 21)$ & $0.11^{\mathrm{a}}$ \\
Biologics & $0(0 / 47)$ & $19(4 / 21)$ & $0.02^{\mathrm{a}}$ \\
CRP (mg/dL) & 0.069 & 0.114 & $0.36^{\mathrm{c}}$ \\
Endoscopic activity (Matts grade) & $1.69(\mathrm{n}=23)$ & $2.14(\mathrm{n}=14)$ & $0.04^{\mathrm{c}}$ \\
\hline E & & & \\
\hline
\end{tabular}

Values are presented as average (age, duration, CRP and endoscopic activity) or percent (number/total number).

${ }^{a}$ Fisher exact test.

${ }^{b}$ Chi-square test.

'Mann-Whitney $U$ test.

5-ASA, 5-aminosalicylic acid; SASP, sulfasalazine.

for the clinical remission group with IBS-like symptoms was 1,090 days, which was shorter as compared to that of the group without IBS-like symptoms ( 1,218 days $)(P<0.01$, logrank test) (Fig. 1). This finding indicated that the presence of IBS-like symptoms might predict UC relapse.

Although the mechanism of IBS-like symptom development is unclear, the presence of low-grade inflammation in colonic mucosa seems to play an important role. ${ }^{5}$ We previously reported that residual low-grade inflammation may influence the presence of IBS-like symptoms in patients with UC in remission. ${ }^{2}$ In the present study, we found that the presence of IBS-like symptoms in association with residual endoscopic activity in patients with UC in clinical remission may influence the relapse of UC. Indeed, the possibility that IBS-like symptoms may indicate an insufficient remission status was corroborated by the shortened relapse-free period of the patients with UC who had IBS-like symptoms in our findings. However, the number of patients who underwent a colonoscopy examination was small in the present study. In addition, we did not measure fecal calprotectin (FC) levels in the enrolled subjects, though increased FC is known to be

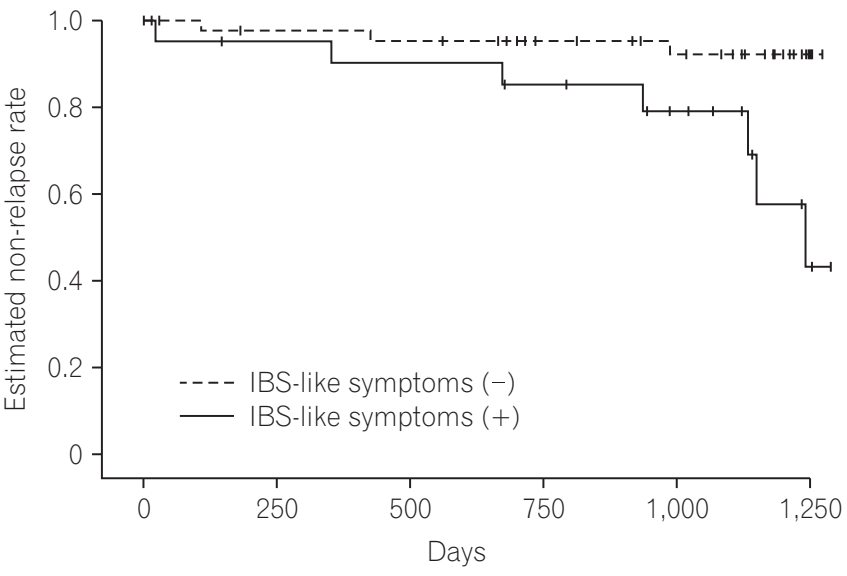

Fig. 1. The relapse-free period of patients with UC in remission with IBS-like symptoms was shorter compared to that of patients with UC without IBS-like symptoms $(P<0.01$, log-rank test).

associated with the presence of IBS-like symptoms. ${ }^{6}$ Further investigations will be necessary to confirm the influence of low-grade colonic inflammation in the presence of IBS-like symptoms using colonoscopy examination results as well as the measurement of FC levels in a larger study cohort.

The present study has several limitations. First, we analyzed the records of patients with UC who had participated in our previous investigation ${ }^{2}$ and follow-up examinations were only conducted for those who were available to be followed. Since the present findings are dependent on the retrospective nature of the study, we were not able to examine an adequate number of subjects. Second, multivariate analysis was not utilized to evaluate the predictors of relapse, thus it cannot be concluded that the presence of IBS-like symptoms in patients with UC without endoscopic activity is actually related to relapse. Additional prospective studies are necessary to elucidate whether the presence of IBS-like symptoms is responsible for UC relapse, irrespective of endoscopic activity.

We concluded that patients with UC with IBS-like symptoms may need to be targeted for treatment, even if they meet the criteria used to define remission, with the aim of long-term remission maintenance.

\section{REFERENCES}

1. Halpin SJ, Ford AC. Prevalence of symptoms meeting criteria for irritable bowel syndrome in inflammatory bowel disease: systematic review and meta-analysis. Am J Gastroenterol 2012;107:1474-1482. 
2. Fukuba N, Ishihara S, Tada Y, et al. Prevalence of irritable bowel syndrome-like symptoms in ulcerative colitis patients with clinical and endoscopic evidence of remission: prospective multicenter study. Scand J Gastroenterol 2014;49:674-680.

3. Rachmilewitz D. Coated mesalazine (5-aminosalicylic acid) versus sulphasalazine in the treatment of active ulcerative colitis: a randomised trial. BMJ 1989;298:82-86.

4. Matts SG. The value of rectal biopsy in the diagnosis of ulcerative colitis. Q J Med 1961;30:393-407.
5. Ishihara S, Tada Y, Fukuba N, et al. Pathogenesis of irritable bowel syndrome: review regarding associated infection and immune activation. Digestion 2013;87:204-211.

6. Keohane J, O’Mahony C, O’Mahony L, O’Mahony S, Quigley EM, Shanahan F. Irritable bowel syndrome-type symptoms in patients with inflammatory bowel disease: a real association or reflection of occult inflammation? Am J Gastroenterol 2010;105:1788-1794. 